\title{
Transcriptomic analysis reveals hub genes and subnetworks related to ROS metabolism in Hylocereus undatus through novel superoxide scavenger trypsin treatment during storage
}

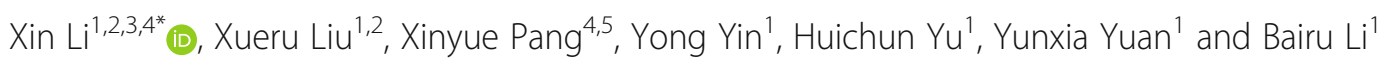

\begin{abstract}
Background: It was demonstrated in our previous research that trypsin scavenges superoxide anions. In this study, the mechanisms of storage quality improvement by trypsin were evaluated in $\mathrm{H}$. undatus.

Results: Trypsin significantly delayed the weight loss and decreased the levels of ROS and membrane lipid peroxidation. Transcriptome profiles of $\mathrm{H}$. undatus treated with trypsin revealed the pathways and regulatory mechanisms of ROS genes that were up- or downregulated following trypsin treatment by gene ontology (GO) and Kyoto Encyclopedia of Genes and Genomes pathway (KEGG) enrichment analyses. The current results showed that through the regulation of the expression of hub redox enzymes, especially thioredoxin-related proteins, trypsin can maintain low levels of endogenous active oxygen species, reduce malondialdehyde content and delay fruit aging. In addition, the results of protein-protein interaction networks suggested that the downregulated NAD(P) $\mathrm{H}$ and lignin pathways might be the key regulatory mechanisms governed by trypsin.
\end{abstract}

Conclusions: Trypsin significantly prolonged the storage life of $H$. undatus through regulatory on the endogenous ROS metabolism. As a new biopreservative, trypsin is highly efficient, safe and economical. Therefore, trypsin possesses technical feasibility for the quality control of fruit storage.

Keywords: GO, KEGG, Hylocereus undatus (H. undatus), Reactive oxygen species (ROS), Protein-protein interaction (PPI), Storage, Trypsin

\footnotetext{
* Correspondence: lixin@haust.edu.cn

${ }^{1}$ College of Food and Bioengineering, Henan University of Science and

Technology, No. 263, Kaiyuan Avenue, Luolong District, Luoyang city 471023,

Henan, China

${ }^{2}$ State Key Laboratory of Cotton Biology, Henan University, Kaifeng 455000,

China

Full list of author information is available at the end of the article
}

C C The Author(s). 2020 Open Access This article is licensed under a Creative Commons Attribution 4.0 International License, which permits use, sharing, adaptation, distribution and reproduction in any medium or format, as long as you give appropriate credit to the original author(s) and the source, provide a link to the Creative Commons licence, and indicate if changes were made. The images or other third party material in this article are included in the article's Creative Commons licence, unless indicated otherwise in a credit line to the material. If material is not included in the article's Creative Commons licence and your intended use is not permitted by statutory regulation or exceeds the permitted use, you will need to obtain permission directly from the copyright holder. To view a copy of this licence, visit http://creativecommons.org/licenses/by/4.0/. The Creative Commons Public Domain Dedication waiver (http://creativecommons.org/publicdomain/zero/1.0/) applies to the data made available in this article, unless otherwise stated in a credit line to the data. 


\section{Background}

Hylocereus undatus ( $H$. undatus) is a perennial climbing cactus plant that is native to tropical areas of Mexico and Central and South America [1]. H. undatus is a nonclimacteric fruit that reaches its best edible quality when harvested ripe, and its quality decreases during storage. The shelf life of fresh $H$. undatus is short. As a newly cultivated crop, few studies have aimed to extend the postharvest quality of this fruit [2].

Disorder of reactive oxygen species (ROS) metabolism and excessive accumulation of ROS causes an increase of membrane lipid peroxidation and leads to fruit spoilage during the process of fruit ripening and decay $[3,4]$. Trypsin is a serine protease and is used as a proteolytic enzyme. It was shown that the presence of trypsin significantly affects the activity of flavonoids in scavenging 2,2-diphenyl-1- picrylhydrazyl (DPPH), 2, 2-azinobis (3ethylbenzo- thiazoline-6-sulphonic acid; ABTS) and hydroxyl radicals [5]. We also reported that trypsin can protect cells by scavenging superoxide anions $\left(\mathrm{O}_{2}^{-}\right)$[6]. The function and mechanisms of the impact of trypsin on the quality of $H$. undatus during storage have not been determined to date.

RNA-seq is a highly efficient technology for gene expression analysis [7, 8]. For plants, RNA-seq has been widely used to identify regulatory mechanisms and screen target genes in the area of phytopathology $[9,10]$. However, few such studies have been performed with respect to gene expression related to postharvest technology of fruits and vegetables. Although the hub genes involved in the biosynthesis of betalain have been identified in $H$. undatus [11], the regulatory mechanisms of postharvest quality of fruits and vegetables have not been elucidated to date.

The analysis of protein-protein interactions (PPI) enables us to further elucidate the biological processes, organization, and action mechanisms of proteins [12]. Cytoscape is an open platform with a series of plugins to make it more visualized and able to perform deep network analysis. A number of the plugins of Cytoscape, such as NetworkAnalyzer, MCODE, or cytoHubba, could be employed to score and rank the nodes or screen modules in the PPI network [13].

In the current study, we investigated the impact of trypsin on the quality and shelf life of $H$. undatus by regulating active oxygen metabolism. The differentially expressed ROS genes (DERGs) of $H$. undatus peel samples were obtained. GO and KEGG enrichment analyses of DERGs were applied, and the PPI network of ROS related genes and subnetwork of DERGs were constructed. The hub genes related to ROS mechanisms of fruit quality improvement by trypsin during storage were further analyzed by Cytoscape with such plugins as cytoHubba and MCODE.

\section{Results}

\section{Effect on storage quality of $H$. undatus}

The $H$. undatus fruits of both groups were in excellent condition at the beginning of storage (Fig. 1). After 159 $h$ of storage, the squamas of the fruits in the control

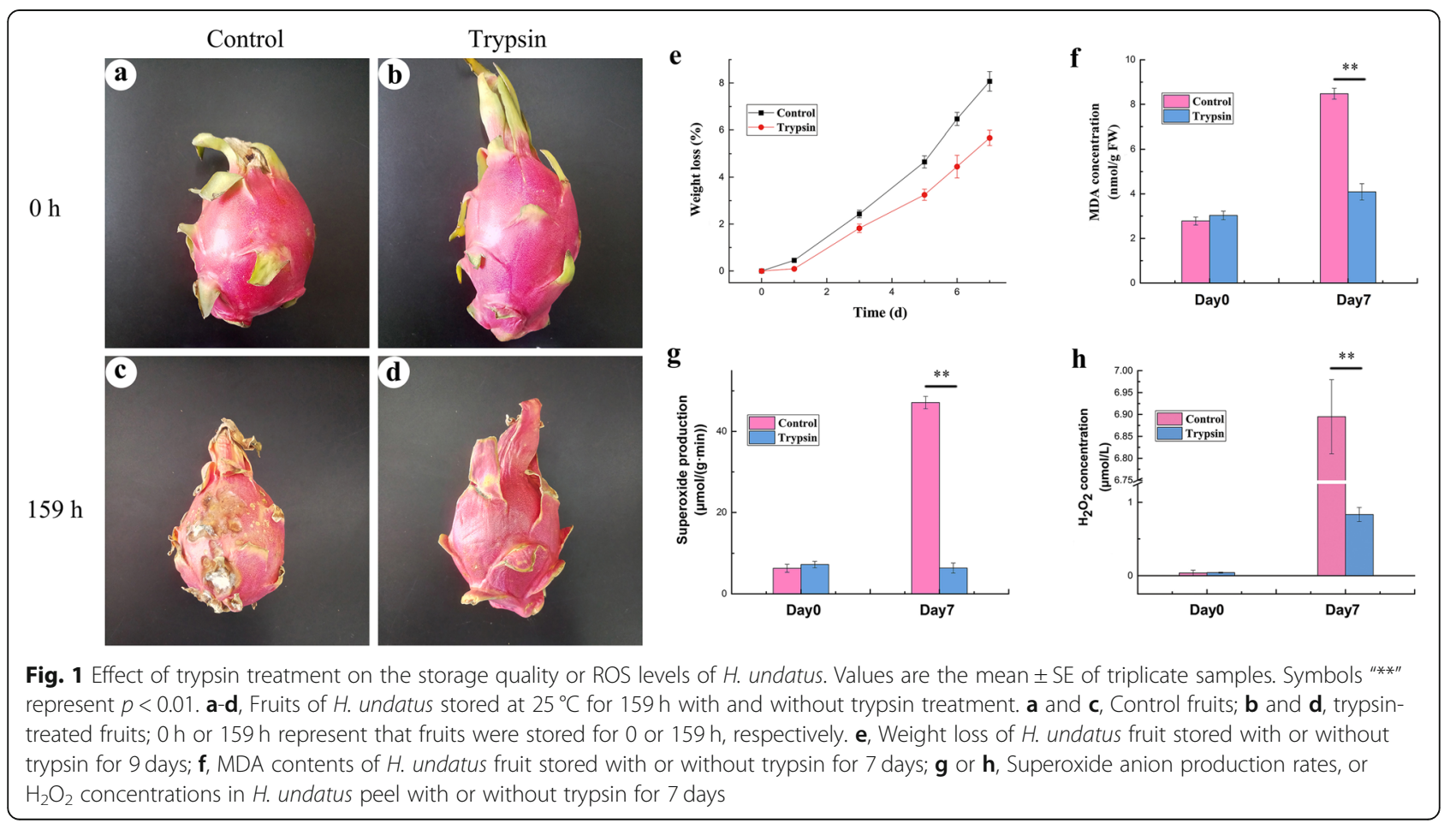


group were completely dry and brittle, the color was dim and the fruit bodies were significantly corrupted with plaque and were inedible (Fig. 1c). In the trypsin group, the squamas were partly dry; however, the peel was bright and clean, and the flesh was edible (Fig. 1d).

The weight loss rate of each group showed a significant upward trend with increasing storage time (Fig. 1e). The fruit in the control group exhibited a relatively large mean decrease in fresh weight of $1.15 \%$ weight loss per day, while the trypsin group showed only a $0.81 \%$ (trypsin) weight loss per day (Fig. 1e). There was a significant difference between the trypsin and control groups $(p<0.01)$.

\section{Impact on the level of cell injury}

To further investigate the preservation mechanism of trypsin, the difference of injury on cells between control and trypsin group was evaluated in $H$. undatus. Figure $1 \mathrm{f}$ showed that the membrane lipid peroxidation in the control group sharply increased by fourfold after 7 days of storage. The increase of malondialdehyde (MDA) was fully impeded by trypsin. There was a highly significant difference between the two groups by the end of the storage period (Day 7) $(p<0.01)$.

\section{Impact on the ROS metabolisms of $H$. undatus}

Excess ROS was the major source of cell injury during storage of fruit. Results showed that the levels of $\mathrm{O}_{2}{ }^{-}$ and $\mathrm{H}_{2} \mathrm{O}_{2}$ in the fruits of the control group increased with storage (Fig. $1 \mathrm{~g}$ and $\mathrm{h}$ ), exhibiting similar trends as did the MDA content. Trypsin entirely inhibited the accumulation of ROS, especially $\mathrm{O}_{2}{ }^{-}$(Fig. $1 \mathrm{~g}$ and $\mathrm{h}$ ).

\section{Transcriptomic analysis}

\section{Sequencing and de novo assembly of Transcriptome}

To further reveal the hub genes and key pathway of ROS regulation by trypsin, transcriptomic data was analyzed in $H$. undatus. The two libraries of the control and trypsin groups produced 50,236,685 and 44,897,144 raw reads, respectively (Table 1 ). The length of a single read was $150 \mathrm{bp}$. From control and trypsin group libraries, 48, 702,393 and 43,456,887 high quality reads were obtained, respectively. Q20 values were 98.31 and $98.33 \%$, respectively. Q30 values were 94.80 and $94.90 \%$, respectively. Also, 224,395 transcripts were composed by highquality reads using Trinity software. The average length was 1086 bp. N50 value was 1982 . The length of transcripts ranged from 201 to $15,462 \mathrm{bp}$. BUSCO score was $64.8 \%$. Figure S1 showed the length distribution of these transcripts. In fact, 78.63 and $72.92 \%$ of transcripts have been mapped, respectively.

\section{Functional annotation and analyses}

Since there was still no reference genome for $H$. undatus, a total of 131,559 transcripts and 86,808 unigenes were blasted against six public databases (Swiss-Prot, NR, COG, Pfam, GO and KEGG) (E value <1e-4). 67,506 (51.31\% of all) transcripts and 31,756 (36.58\% of all) unigenes were annotated using these databases (Fig. S2, Table S1 and S2). Based on COG and NOG classifications, 3191 or 2755 unigenes and 8288 or 6677 transcripts were assigned into 24 functional groups, respectively (Fig. S3 and Table S3). The number of transcripts was much higher than that of unigenes. Each unigene was spliced by one or more transcripts. All of these unigenes belonging to three different categories, including biological process (BP), molecular function (MF), or cellular component (CC), have been classified (Fig. S4a).

The main functions were gathered in "binding" and "catalytic activity" on level 2 of molecular function classification (Fig. S4b). In the category of biological processes, they were focused on "organic substance metabolic process" (7758 unigenes, 15.98\%), "primary metabolic process" (5442 unigenes, 15.12\%) and "cellular metabolic process" (5247 unigenes, 14.54\%) (Fig. S4c). In the cellular component, cell part (13.01\%) and membrane part (12.35\%) were the major parts (Fig. S4d). 7943 unigenes were assigned to $20 \mathrm{~s}$ categories belonging to 6 first categories of KEGG pathways (E-value: 1e-4; Identity: 0; Similarity: 0) (Fig. S5 and Table S4).

\section{Analysis of differentially expressed genes with trypsin treatment}

A total of 31,756 unigenes were identified and quantified from 86,808 genes (Table S1). The expression levels of these genes have been concluded by using a volcano plot (Fig. 2a and Table S5). The total number of different unigenes identified was 1703, including 934 upregulated unigenes (red points) and 769 downregulated unigenes (green points), $(p<0.05$, fold change $(\mathrm{FC})>1)$ in the control and treatment groups (Table S5). On the other hand, a total of 1117 ROS related genes (orange circle) were determined, including 434 upregulated unigenes (yellow circle) and 465 downregulated unigenes (purple circle). Among the 1117 ROS related genes, 85 genes (green circle) involved in 1703 genes (blue circle) were

Table 1 Summary of the sequencing data of $H$. undatus

\begin{tabular}{llllllllll}
\hline Sample & Raw reads & Raw bases & Clean reads & Clean bases & Error rate (\%) & Q20 (\%) & Q30 (\%) & GC content (\%) & Mapped ratio (\%) \\
\hline Control & $50,236,685$ & $7,535,502,700$ & $48,702,393$ & $6,604,345,574$ & 0.013 & 98.31 & 94.80 & 46.87 & 78.63 \\
Trypsin & $44,897,144$ & $6,734,571,600$ & $43,456,887$ & $5,841,023,572$ & 0.012 & 98.33 & 94.90 & 45.19 & 72.92 \\
\hline
\end{tabular}



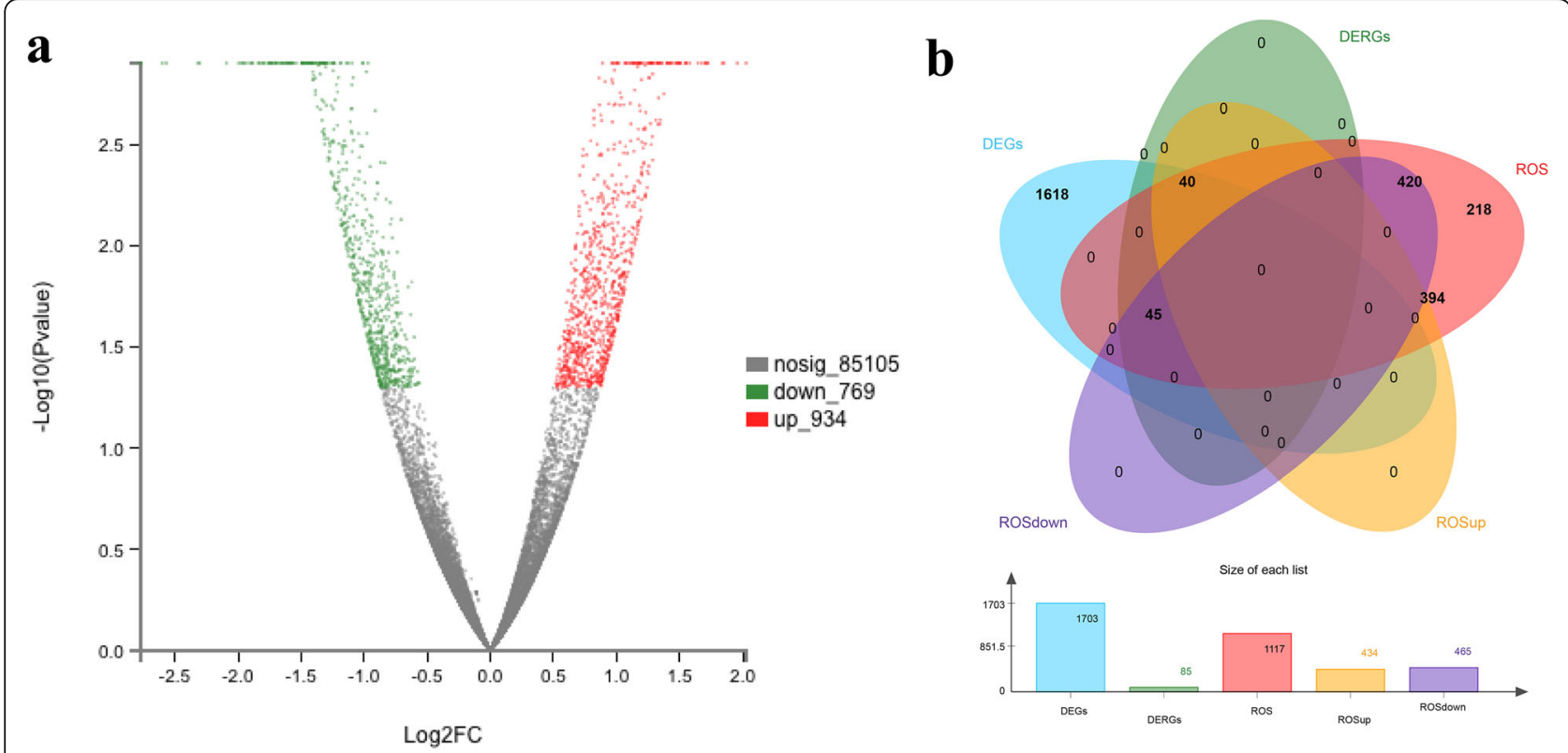

Fig. 2 Analysis of differentially expressed genes with trypsin. a Volcano plot of significant differences in gene expression between control and trypsin groups; b Venn diagram representation of all 1703 differentially expressed genes (DEGs), upregulated genes, downregulated genes, and all of the 1117 ROS related genes (ROSup, ROSdown, and ROS), and 85 DERGs identified in the trypsin treatment group

expressed to a significantly different extent (Fig. $2 \mathrm{~b}$ and Table S6).

\section{GO enrichment analyses}

The biological functions of the patterns up- or downregulated by trypsin treatment were analyzed by gene ontology (GO)-based enrichment. The top $10 \mathrm{GO}$ terms in the two expression patterns were shown in Table 2 $\left(\mathrm{FDR}<10^{-6}\right)$. The upregulated enriched GO terms were "oxidoreductase activity, acting on single donors with incorporation of molecular oxygen (GO: 0016701)" and "oxidoreductase activity (GO:0016491)." On the other hand, besides more oxidation/reduction GO terms, "hydrogen peroxide catabolic process (GO: 0042744)," "peroxidase activity (GO: 0004601)," "oxidoreductase activity, acting on $\operatorname{NAD}(\mathrm{P}) \mathrm{H}$, oxygen as acceptor (GO: 0050664)," and so on, antioxidant or catabolic GO terms, including "peroxidase activity," "antioxidant activity" or "catalytic activity" were presented in the downregulated pattern (Table S7).

The major ROS related pathways involved in trypsin regulation can be summarized into a schematic representation (Fig. 3). GO terms are related to one another in a directed acyclic graph (DAG), where more detailed

Table 2 Top $10 \mathrm{GO}$ terms related to ROS enriched $\left(\mathrm{FDR}<10^{-6}\right.$ ) by trypsin

\begin{tabular}{|c|c|c|c|c|c|c|}
\hline Pattern & Number & $\mathrm{GO} I \mathrm{D}$ & $\begin{array}{l}\text { Term } \\
\text { Type }\end{array}$ & Description & $p$-value & $\mathrm{FDR}^{\mathrm{a}}$ \\
\hline \multirow[t]{2}{*}{ Upregulation } & 8 & GO:0016701 & MF & $\begin{array}{l}\text { oxidoreductase activity, acting on single donors with incorporation of } \\
\text { molecular oxygen }\end{array}$ & $2.01 \mathrm{E}-12$ & $1.11 \mathrm{E}-08$ \\
\hline & 25 & GO:0016491 & MF & oxidoreductase activity & $3.79 \mathrm{E}-11$ & 1.05E-07 \\
\hline \multirow[t]{8}{*}{ Downregulation } & 6 & GO:0042744 & $\mathrm{BP}$ & hydrogen peroxide catabolic process & $7.85 \mathrm{E}-10$ & 6.07E-07 \\
\hline & 6 & GO:0042743 & $\mathrm{BP}$ & hydrogen peroxide metabolic process & $8.80 \mathrm{E}-10$ & 6.07E-07 \\
\hline & 11 & GO:0004601 & MF & peroxidase activity & $4.05 \mathrm{E}-12$ & 1.77E-08 \\
\hline & 11 & GO:0016684 & MF & oxidoreductase activity, acting on peroxide as acceptor & $6.40 \mathrm{E}-12$ & 1.77E-08 \\
\hline & 11 & GO:0016209 & MF & antioxidant activity & $2.75 \mathrm{E}-11$ & 5.07E-08 \\
\hline & 5 & GO:0050664 & MF & oxidoreductase activity, acting on $\mathrm{NAD}(\mathrm{P}) \mathrm{H}$, oxygen as acceptor & $5.88 \mathrm{E}-11$ & 8.06E-08 \\
\hline & 28 & GO:0016491 & MF & oxidoreductase activity & 7.30E-11 & 8.06E-08 \\
\hline & 31 & GO:0003824 & MF & catalytic activity & $1.80 \mathrm{E}-10$ & $1.65 \mathrm{E}-07$ \\
\hline
\end{tabular}




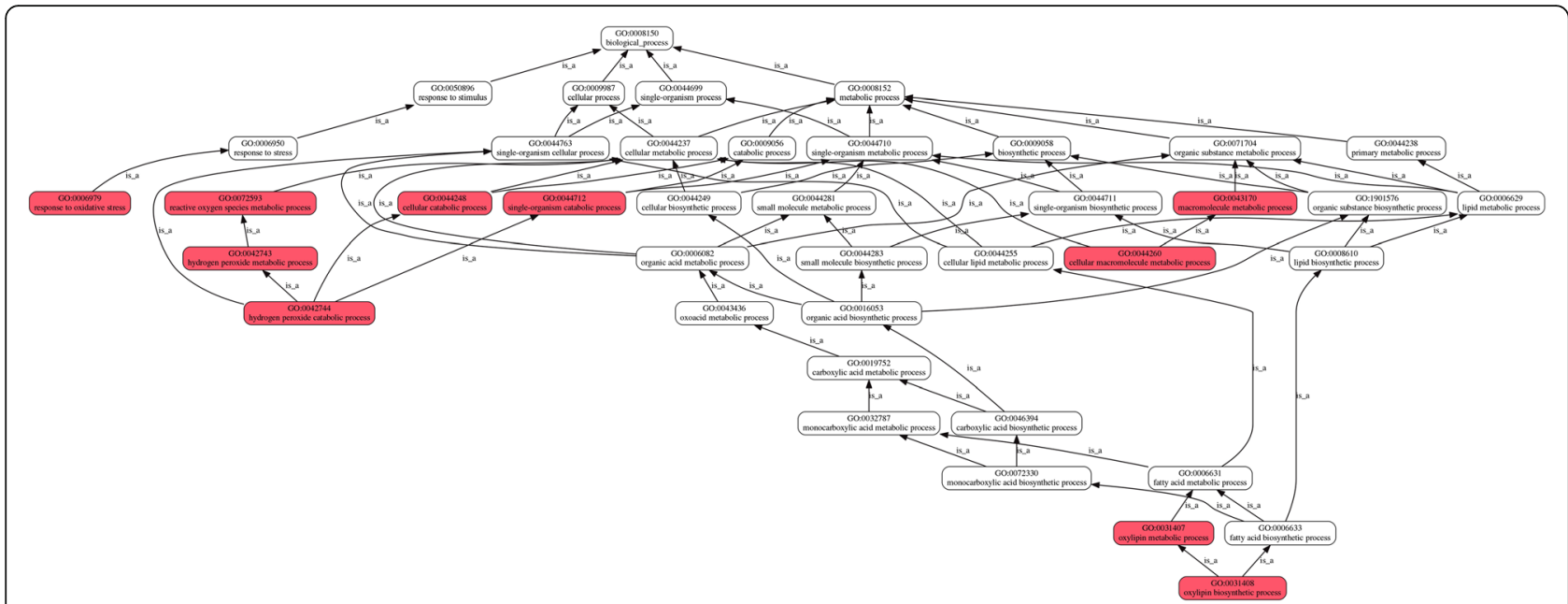

Fig. 3 Relationships between GO terms in a Directed Acyclic Graph (DAG). Red to white colors represent decreasing significance levels (Red is the most, while white is the least significant). The figure illustrates a subset of the molecular function DAGs for the oxylipin biosynthetic process (GO:0031408), hydrogen peroxide catabolic process (GO:0042744) and response to oxidative stress (GO:0006979). Arrows indicate relationships of the is_a type. The ancestors of GO:0031408, GO:0042744 or GO:0006979 are highlighted back to the root of the biological process ontology via arrows

terms are described as children of more general terms. For example, the GO biological process "hydrogen peroxide catabolic process (GO:0042744)" is a child of four terms: "single-organism cellular process (GO:0044763)," "hydrogen peroxide metabolic process (GO:0042743)," "cellular catabolic process (GO:0044248)," and "singleorganism catabolic process (GO:0044712)." The GO biological process "oxylipin biosynthetic process (GO: 0031408)" is a child of two terms: "oxylipin metabolic process (GO:0031407)" and "fatty acid biosynthetic process (GO:0006633)." These in turn have parent terms, as shown in Figure 3, tracing back to the ultimate ancestor, biological process (GO:0008150), the root of the molecular function ontology. In addition, $\mathrm{H}_{2} \mathrm{O}_{2}$ catabolic metabolism was only downregulated (Fig. S6). Cell redox homeostasis was shown to be an upregulated GO process (Fig. S7).

\section{KEGG pathway analyses}

The top 9 enriched pathways were shown in Table 3 (FDR $<0.05$ ). With trypsin, downregulated ROS related genes were enriched in several pathways, including the "phenylpropanoid biosynthesis pathway (map 00940)," which is associated with fatty acid biosynthesis, as shown in the GO analysis section, "MAPK signaling pathway - plant (map 04016)," which involves a series of defense responses induced by ROS, and "Plant-pathogen interaction (map 04626)," which highly focus on the hypersensitive response (HR) induced by ROS. On the other hand, "Linoleic acid metabolism (map 00591)," "Photosynthesis (map 00195)," "Ascorbate and aldarate metabolism (map
00053)," and "Porphyrin and chlorophyll metabolism (map 00860)" were significantly upregulated.

\section{PPI networks of DERGs}

In total, we obtained 85 DERGs (FDR $<0.01,40$ upregulated and 45 downregulated) among 1117 ROS related genes, including 434 upregulated genes and 465 downregulated genes (Table $\mathrm{S} 8$ ).

The PPI subnetwork of total ROS genes was composed of 404 nodes and the first 2000 edges, containing 40 DERGs (Fig. 4 and Table S9). The upregulated ROS gene PPI subnetwork contained 278 nodes and the first 2000 edges, including 29 upregulated DERGs (Fig. S8a and Table S10). The downregulated antioxidant gene PPI subnetwork contained 288 nodes and the first 2000 edges (interactions), including 10 downregulated DERGs (Fig. S8b and Table S11).

Further, the Cytoscape plugin "MCODE" was layered on the PPI network. Twenty clusters were obtained (Table S12). Nodes belonging to the top 6 clusters were labeled by different colors in the PPI network (Fig. 4). The top 6 clusters analyzed by the CytoHubba plugin of Cytoscape were then shown in Figure 5. The central nodes of each cluster were shown in Table 4 .

Furthermore, all of the DERGs were selected to construct 3 new PPI networks, including total (60 nodes, 255 edges) (Fig. 6a), upregulated (30 nodes, 81 edges) (Fig. S9a), and downregulated (28 nodes, 62 edges) DERGs subnetworks (Fig. S9b). Three clusters were constructed by MCODE as shown in Figure $6 \mathrm{c}, \mathrm{d}$ and e.

Based on the calculation of CytoHubba plugin, because the screen results of either Density of Maximum 
Table 3 Pathways related to ROS enriched (FDR < 0.05) by trypsin

\begin{tabular}{llllll}
\hline Pattern & Number & KO ID & Term & $p_{\text {-value }}^{*}$ & FDR $^{\text {a }}$ \\
\hline Upregulation & 3 & map 00591 & Linoleic acid metabolism & $1.56 \mathrm{E}-05$ & 0.00034 \\
& 3 & map 00195 & Photosynthesis & 0.00017 & 0.0018 \\
& 2 & map 00053 & Ascorbate and aldarate metabolism & 0.0084 & 0.0065 \\
Downregulation & 2 & map 00860 & Porphyrin and chlorophyll metabolism & $1.29 \mathrm{E}-10$ & 5.047 \\
& 9 & map 00940 & Phenylpropanoid biosynthesis & $5.52 \mathrm{E}-06$ & $2.06 \mathrm{E}-09$ \\
& 6 & map 04016 & MAPK signaling pathway - plant & $1.34 \mathrm{E}-05$ & $7.41 \mathrm{E}-05$ \\
& 6 & map 04626 & Plant-pathogen interaction & 0.0020 & 0.0080 \\
& 2 & map 00073 & Cutin, suberin and wax biosynthesis & 0.010 & 0.033 \\
\hline
\end{tabular}

${ }^{*} p$-values were calculated using Fisher's test

${ }^{a}$ FDR corrections were calculated using the Benjamini-Hochberg procedure

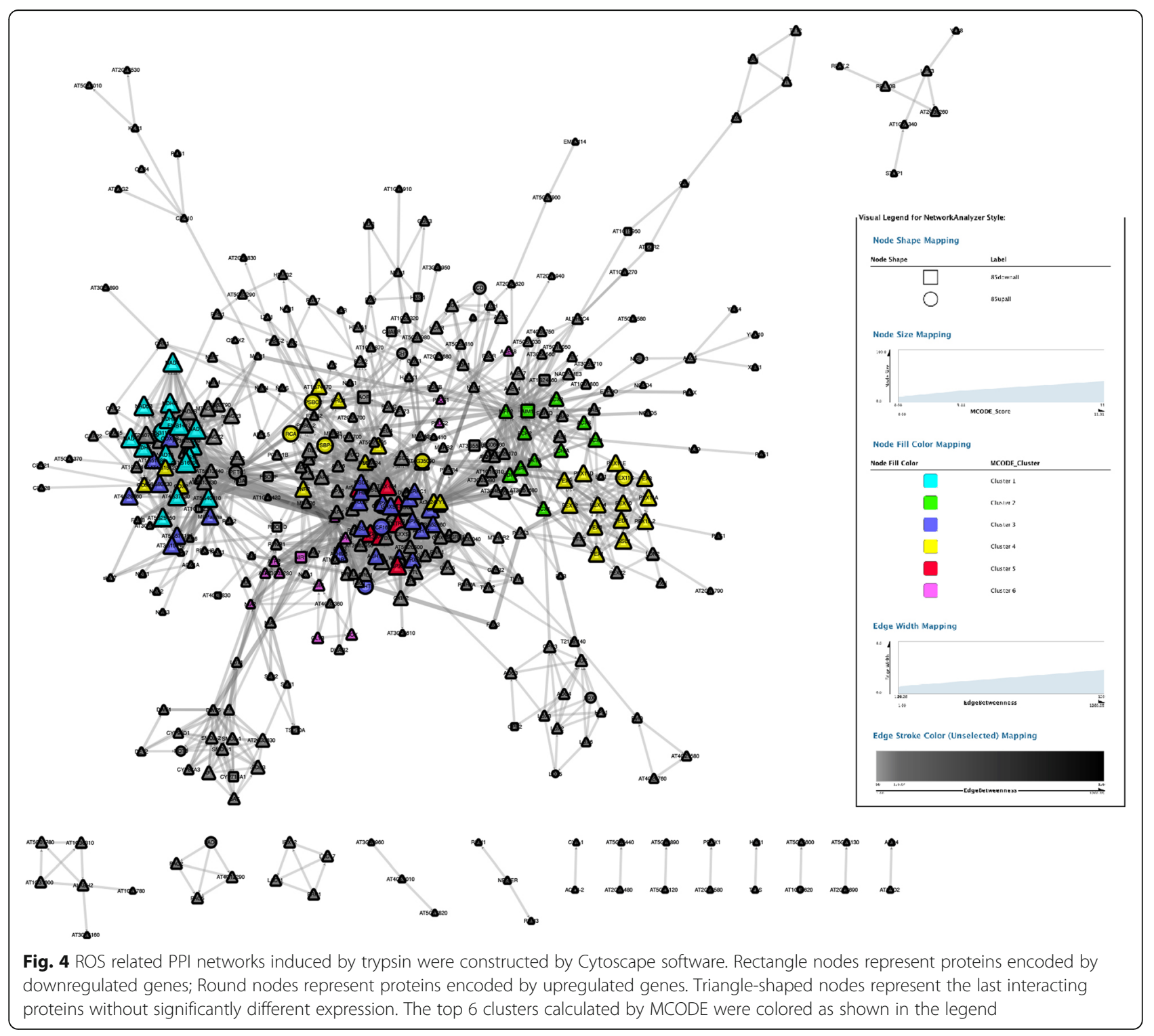




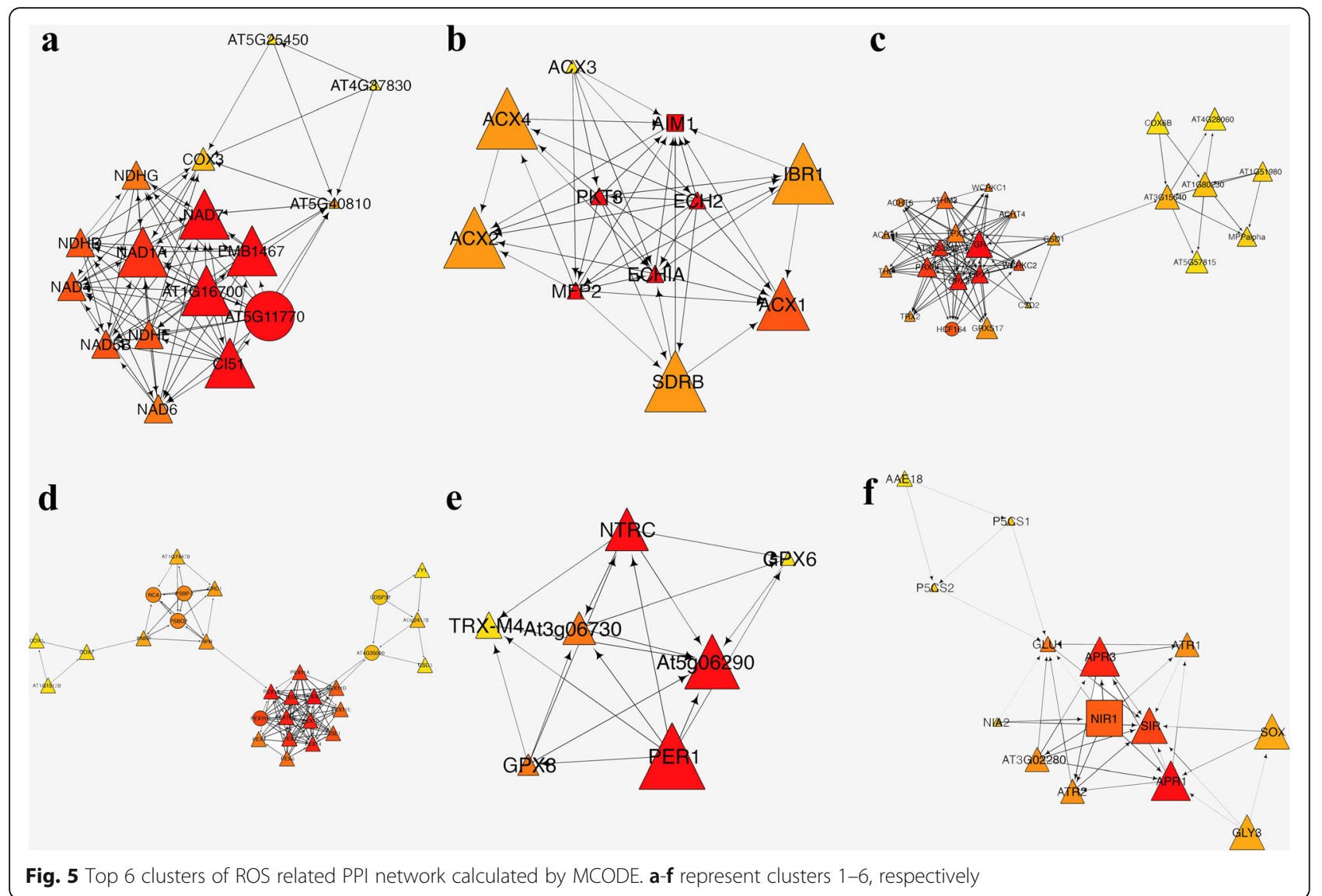

Neighborhood Component (DMNC) or Maximum Neighborhood Component (MNC) did not match well to the other methods, hub genes were determined by overlapping the genes according to 4 ranked methods, including Maximal Clique Centrality (MCC), Degree, Closeness and Betweenness in cytoHubba (Table S13). Ten hub genes were discovered, including 7 upregulated and 3 downregulated genes (Fig. $6 \mathrm{~b}$ and $\mathrm{f}$ ).

\section{Topological properties of networks}

The node degree distributions of the total, upregulated, and downregulated ROS related gene subnetworks followed power law fit distributions $\left(R^{2}=0.798,0.765\right.$, and 0.762 , respectively) (Fig. S10). The subnetwork topological parameters, including network centralization clustering coefficient, and so on, were shown in Table 5.

The subnetworks of DERGs, except the downregulated DERGs subnetwork $\left(R^{2}=0.001\right)$, were characterized as scale-free networks, even though they were much weaker than ROS related genes networks, which approximately followed power law fit distributions, with $R^{2}=0.412$ and 0.422 , respectively (Fig. S10 and Table 5). Since the DERGs were chosen from the ROS related genes network, either the centralization or the density of the total DERGs network was much higher (0.395 and 0.144 , respectively) than that of the total ROS related genes network ( 0.150 and 0.025 , respectively). Finally, the correlation of hub network was decreased to 0.499 $\left(R^{2}=0.268\right)$.

Table 4 Central nodes of top 6 clusters of ROS related PPI subnetwork of H. undatus

\begin{tabular}{llll}
\hline Cluster & Central nodes & Score of clusters & Score of each node \\
\hline 1 & AT1G16700; NAD7; EMB1467; AT5G11770; CI51 & 322,680 \\
2 & PKT3; ECHIA; MFP2; AIM1; ECH2 & 10.533 & 2280 \\
3 & GR & 8.600 & 344 \\
4 & PEX5 & 7.750 & 171,362 \\
5 & At5g06290; PER1; NTRC & 7.571 & 72 \\
6 & APR1 & 6.000 & 42 \\
\hline
\end{tabular}




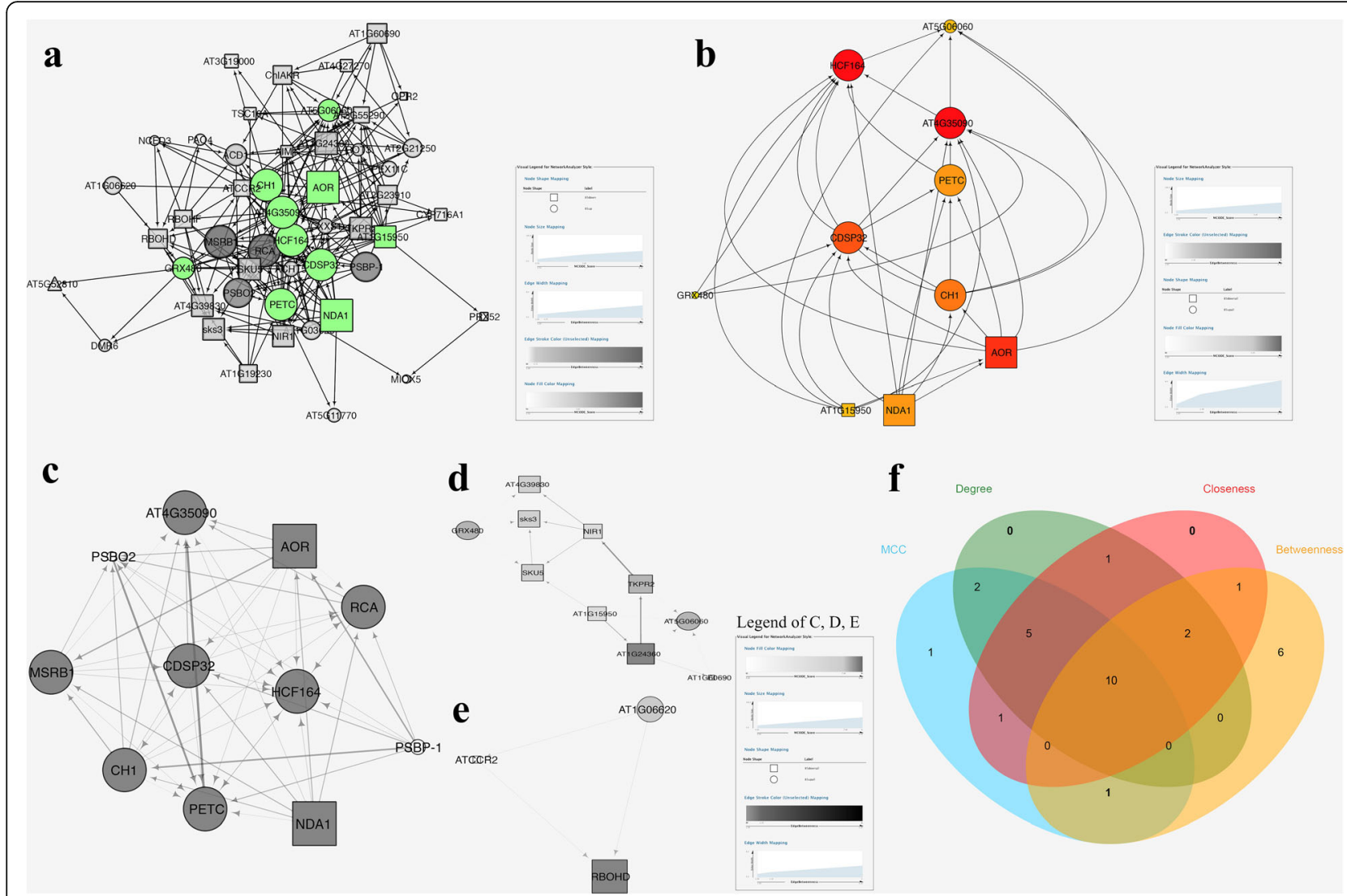

Fig. 6 Analysis of hub genes of DERGs. The shape setting of nodes was the same as in Figure 4. a, Subnetwork of DERGs. The top 10 nodes of cytoHubba were colored; $\mathbf{b}$, Hierarchical layout of 10 hubs; $\mathbf{c}-\mathbf{e}$, Clusters 1-3 of DERGs by MCODE; $\mathbf{f}$, Overlapping DERGs among the four methods according to cytoHubba

Table 5 Topological parameters of the ROS related genes, DERGs and Hub genes of the PPI subnetwork of $H$. undatus impacted by trypsin

\begin{tabular}{|c|c|c|c|c|c|c|c|c|}
\hline PPI subnetwork & $y=\beta x^{a}$ & $R^{2}$ & Correlation & $\begin{array}{l}\text { Clustering } \\
\text { coefficient }\end{array}$ & $\begin{array}{l}\text { Network } \\
\text { centralization }\end{array}$ & $\begin{array}{l}\text { Network } \\
\text { density }\end{array}$ & $\begin{array}{l}\text { Num. of } \\
\text { nodes }\end{array}$ & $\begin{array}{l}\text { Characteristic path } \\
\text { length }\end{array}$ \\
\hline \multicolumn{9}{|c|}{ ROS related genes network } \\
\hline Total ROS & $y=137.73 x^{-1.213}$ & 0.798 & 0.891 & 0.381 & 0.150 & 0.025 & 404 & 3.920 \\
\hline Upregulated ROS & $y=52.493 x^{-0.889}$ & 0.765 & 0.853 & 0.413 & 0.286 & 0.052 & 278 & 2.839 \\
\hline $\begin{array}{l}\text { Downregulated } \\
\text { ROS }\end{array}$ & $y=60.805 x^{-0.931}$ & 0.762 & 0.733 & 0.353 & 0.299 & 0.048 & 288 & 2.748 \\
\hline \multicolumn{9}{|c|}{ DERGs of ROS subnetwork } \\
\hline Total DERGs & $y=7.431 x^{-0.521}$ & 0.412 & 0.582 & 0.361 & 0.395 & 0.144 & 60 & 2.397 \\
\hline $\begin{array}{l}\text { Upregulated } \\
\text { DERGs }\end{array}$ & $y=4.697 x^{-0.532}$ & 0.422 & 0.807 & 0.354 & 0.355 & 0.186 & 30 & 2.448 \\
\hline $\begin{array}{l}\text { Downregulated } \\
\text { DERGs }\end{array}$ & $y=3.289 x^{-0.026}$ & 0.001 & -0.021 & 0.239 & 0.222 & 0.164 & 28 & 2.437 \\
\hline \multicolumn{9}{|l|}{ Hubs subnetwork } \\
\hline Total 10 hubs & $y=0.688 x^{0.468}$ & 0.268 & 0.499 & 0.849 & 0.333 & 0.733 & 10 & 1.267 \\
\hline
\end{tabular}




\section{Accuracy of RNA-Seq data verification by RT-qPCR}

The accuracy of the RNA-Seq data of ten hub genes of DERGs involved in ROS metabolism were verified by reverse transcription quantitative PCR (RT-qPCR) (Fig. S11). The IDs, NR description, $\log _{2} \mathrm{FC}(\mathrm{E} / \mathrm{C}), p$ value, FDR, and primers of the 10 hub genes are shown in Table 6 and Table S16.

\section{Discussion}

Trypsin treatment alone can already significantly reduce the loss of water, impede the dehydration and improve the fruit quality. MDA is considered to represent the degree of cell membrane lipid peroxidation, since it is a marker of membrane lipid peroxidation [14]. The result of MDA indicates that trypsin can significantly decrease the MDA content, which represents the lipid peroxidation of the cell membrane, thereby significantly slowing the cell damage.

In the process of maturity or decline, the disruption of balance between the generation and scavenging of ROS causes the accumulation of ROS. A high concentration of ROS such as $\mathrm{O}_{2}{ }^{-}$and $\mathrm{H}_{2} \mathrm{O}_{2}$ can cause lipid peroxidation, which is the main cause of membrane damage [15]. As expected, the novel superoxide scavenger trypsin entirely inhibited the accumulation of ROS, especially $\mathrm{O}_{2}{ }^{-}$.
However, how can trypsin perform ROS regulation? Which genes were impacted by trypsin during storage? The transcriptomic analysis was used to reveal the hub genes regulating ROS metabolism through trypsin treatment.

Analyzed from transcriptomic profile, a total of 1117 ROS related genes were determined, including 434 upregulated unigenes and 465 downregulated unigenes. Among the 1117 ROS related genes, 85 genes were expressed to a significantly different extent.

The biological functions of the patterns up- or downregulated by trypsin treatment were analyzed by gene ontology (GO)-based enrichment. Lots of oxidation/reduction GO terms were enriched. The major ROS related pathways involved in trypsin regulation were summarized by DAGs (Fig. 3). The DAGs indicated that the $\mathrm{H}_{2} \mathrm{O}_{2}$ catabolic metabolism and oxylipin biosynthesis are key processes of trypsin regulatory mechanisms during $H$. undatus storage.

Next, to illustrate the pathways involved in the trypsin responsive patterns, the KEGG pathways were enriched. Important physiological metabolic processes, such as photosynthesis, porphyrin and chlorophyll metabolism, were induced, while defense responses were impeded by trypsin. In the top 2 pathways regulated by trypsin, 11 and 7 genes were involved in "Phenylpropanoid

Table 6 Primer sequences used in RT-qPCR

\begin{tabular}{|c|c|c|}
\hline Name & Gene ID & Primer \\
\hline \multirow[t]{2}{*}{$\beta$-actin } & & Forward: 5'-TCTGCTGAGCGAGAAAT-3' \\
\hline & & Reward: 5'-AGCCACCACTAAGAACAAT-3' \\
\hline \multirow[t]{2}{*}{ HCF164 } & TRINITY_DN39264_C0_g2 & Forward: 5'-GCGAATGATAATCCAAGCCAAGC -3' \\
\hline & & Reward: 5'-TATGATACCGCCAAGGCAGACAG -3' \\
\hline \multirow[t]{2}{*}{ CDSP32 } & TRINITY_DN42075_C0_g1 & Forward: 5'-TTCGCTTCTCCTTTCTCCCTTGC-3' \\
\hline & & Reward: 5'-TGCTGTGGACTITCTGGACCCTC-3' \\
\hline \multirow[t]{2}{*}{ AOR } & TRINITY_DN44827_C0_g2 & Forward: 5'-GAGTACACGGCTGCTGAAGAAAG-3' \\
\hline & & Reward: 5'-GGCACCACCAAGAATAAGGATAG-3' \\
\hline \multirow[t]{2}{*}{$\mathrm{CH} 1$} & TRINITY_DN40044_C0_g1 & Forward: 5'-AGGTTAGAGGCAACATTGGAGTC-3' \\
\hline & & Reward: 5'-ATGGAGCATCATTATGGTGAGAA-3' \\
\hline \multirow[t]{2}{*}{ PETC } & TRINITY_DN41555_c0_g1 & Forward: 5'-CCCATCAACAGCGGTGGCTAAAC-3' \\
\hline & & Reward: 5'-GGGACGAAGAAGGCAGCGTAAGG-3' \\
\hline \multirow[t]{2}{*}{ AT4G35090 } & TRINITY_DN52071_C2_g1 & Forward: 5'-GCAGCCCTGAAACCATCCGTGAC-3' \\
\hline & & Reward: 5'-CCCACATCGTCGAAGAGCCAACC-3' \\
\hline \multirow[t]{2}{*}{ NDA1 } & TRINITY_DN41480_C0_g1 & Forward: 5'-GAGAGAGAGCCAATATCCTATGAAGCATCC-3' \\
\hline & & Reward: 5'-GGATGCTTCATAGGATATTGGCTCTCTCTC-3' \\
\hline \multirow[t]{2}{*}{ AT5G06060 } & TRINITY_DN39500_C0_g1 & Forward: 5'-GGGTCTCAGGAAAGAAACAGTAA-3' \\
\hline & & Reward: 5'-GCTCCATAAATAGGACCAGCATTA-3' \\
\hline \multirow[t]{2}{*}{ AT1G15950 } & TRINITY_DN6609_c0_g1 & Forward: 5'-AAAGAATGCCCATTTGAGGGAGC-3' \\
\hline & & Reward: 5'-TITGTGCCGTTTACTGCTGGTTC-3' \\
\hline \multirow[t]{2}{*}{ GRX480 } & TRINITY_DN13090_C0_g1 & Forward: 5'-AACCACCTTACCAAGAGCTTCCC-3' \\
\hline & & Reward: 5'-CCAATAACAAACGCCTGACAACAT-3' \\
\hline
\end{tabular}


biosynthesis (map 00940)" and "MAPK signaling pathway- plant (map 04016)". As we know, peroxidase (POD) is the key enzyme of the last process of lignin synthesis [16, 17]. In map 00940, Guaiacyl lignin, 5hydroxy-gualacyl lignin, syringyl lignin and p-hydroxyphenyl lignin were regulated by POD (Fig. S12). In map 04016, results showed that respiratory burst oxidase (RbohD) was regulated to maintenance the homeostacis of ROS, and stress-tolerant response was also induced which due to the significantly activated catalase (CAT) (Fig. S13). These observations were consistent with our previous results [18]. The results of KEGG indicated that, as a novel superoxide scavenger, trypsin regulated antioxidant system of pitaya and exhibited protection of pitaya during storage.

It is essential to explore the potential ROS regulatory mechanisms of trypsin by the exposition of the DERGs; the investigation on the PPI networks would promote the functional research on DERGs induced by trypsin. The PPI network of total, upregulated, or downregulated ROS genes were constructed. These three subnetworks indicated that trypsin treatment greatly disturbed the PPI network in $H$. undatus. The biological consequences were magnified by hundreds of proteins interacting with DERGs.

Further, the Cytoscape plugin "MCODE" and "CytoHubba" were layered on the PPI network to illustrate subnetworks and screen hub genes. Among the obtained 10 hub nodes, 7 nodes, including HCF164 (Thioredoxin-like protein), AT4G35090 (Catalase), PETC, and so on, were clustered in module 1 of the DERGs PPI network; the other 3 nodes including AT5G06060 (NAD(P)-binding Rossmann-fold superfamily protein), GRX480 (Thioredoxin superfamily protein), and AT1G15950 (CinnamoylCoA reductase) were clustered in module 2 of the DERGs PPI network (Table S14 and S15). In addition, only HCF164 was clustered in the top 6 modules (cluster 3) of all ROS related PPI networks (Figs. 5 and 6). Most of the hubs were thioredoxin related proteins. This result indicated that the mechanisms of trypsin regulation of ROS are closely associated with sulfur metabolism.

Furthermore, the hub of AT1G15950 (CinnamoylCoA reductase) suggested that the quality of $H$. undatus storage was associated with lignin metabolism, which was also shown in the DAG of GO analysis (Fig. 3). From the information of the arrow directions, either NDA1 (Internal NAD(P) H dehydrogenase in mitochondria) or AOR (NADPH-dependent alkenal/one oxidoreductase, chloroplastic) is the upstream gene of AT5G06060 (Fig. 6). This pathway is dependent on the redox of $\mathrm{NAD}(\mathrm{P}) \mathrm{H}$, which is the main source of $\mathrm{O}_{2}{ }^{-}$. These results strongly indicated that the downregulated $\mathrm{NAD}(\mathrm{P}) \mathrm{H}$ and lignin pathways might be the key regulatory mechanisms of trypsin, the superoxide scavenger, on the quality improvement of $H$. undatus during storage. While, there still lots of question unclear. For example, who is the transcriptional regulation factor primarily induced by trypsin? Further works are needed to investigate the regulatory mechanisms of trypsin on lignin synthesis.

The correlation of the distribution of node degrees of a PPI network to the power law distribution is a judgment standard for scale-free networks. This property distinguishes the PPI network from random networks [19]. The node degree distributions of the total, upregulated, and downregulated ROS related gene subnetworks followed power law fit distributions. This suggested that these three PPI subnetworks were true cellular complex biological scale-free networks. These results also showed that a few protein nodes serve as hubs with links to other protein nodes [19].

As expected, the correlation of hub network was decreased with the decrease of nodes of total ROS, DERGs, and hub networks (Table 5). This indicates that the hub genes are highlighted in a larger network and that our PPI networks are reliable.

The accuracy of the RNA-Seq data of these hub genes were verified by RT-qPCR. Expression changes of these 10 genes were consistent with the RNA-Seq data. This showed that RNA-Seq data are credible.

\section{Conclusions}

Trypsin treatment significantly reduced the accumulation of ROS, including $\mathrm{O}_{2}{ }^{-}$and $\mathrm{H}_{2} \mathrm{O}_{2}$, in $H$. undatus during storage, impeded membrane lipid peroxidation, and prolonged the storage life of $H$. undatus. Transcriptomic analysis revealed 10 hub genes regulated by trypsin involved in $H$. undatus quality improvement during storage. PPI network analysis suggested that the downregulated $\mathrm{NAD}(\mathrm{P}) \mathrm{H}$ and lignin pathways might represent the key regulatory mechanisms of trypsin. As a new biopreservative, trypsin is highly efficient, safe and economical. Therefore, trypsin possesses technical feasibility for the quality control of fruit storage.

\section{Methods \\ Main materials}

H. undatus (Vietnam No. 1 cultivar, white pulp) was harvested from Ruyang county in Henan Province, China. The plant was identified by Prof. Zhaoyong Shi (College of Agriculture, Henan University of Science and Technology, Luoyang, China) and the voucher specimens (No. Hu-20,160,923) have been deposited in our laboratory. Fruits without mechanical damage and with uniform color, size and number of scales were chosen for the study. Trypsin (bovine, 500 units/mg, crystalline) was purchased from Ameresco (Solon, OH, USA). 


\section{$H$. undatus treatment methods}

Trypsin was brushed evenly for $80 \mathrm{~s}$ onto the peels of 15 $H$. undatus fruits as the trypsin group. The fruits of $H$. undatus of the control group were treated in the same conditions with PBS buffer. The fruits were then placed in an incubator $\left(25^{\circ} \mathrm{C}, 85 \%\right.$ relative humidity), and their physical and chemical indices (weight loss, MDA, etc.) were periodically measured. The optimal concentration of trypsin $\left(2.41 \times 10^{-6} \mathrm{~mol} / \mathrm{L}\right)$ was determined in our pre-experiment and used for the current study.

\section{Library construction and Illumina RNA-sequencing}

Total RNA was extracted from two groups of $H$. undatus peels (with or without trypsin). The peels of each group were taken from 15 fruits. Transcriptome libraries for RNA-seq were constructed from $5 \mu \mathrm{g}$ samples of RNA ( $\geq 100 \mathrm{ng} / \mu \mathrm{L})$ using the Truseq ${ }^{\mathrm{TM}}$ RNA sample preparation kit from Illumina (San Diego, CA). Libraries were constructed and sequenced with the Illumina HiSeq xten $(2 \times 150$ bp read length) by the Tolo Biotech company of China. All data were uploaded to the ISanger cloud platform and analyzed as described below.

\section{De novo assembly and annotation}

The raw paired end reads were trimmed and quality controlled by SeqPrep (https://github.com/jstjohn/SeqPrep) and Sickle (https://github.com/najoshi/sickle) with default parameters. Then clean data from the samples were used to do de novo assembly with Trinity (http:// trinityrnaseq.sourceforge.net/) [20]. All the assembled transcripts were searched against the NCBI protein nonredundant (NR), String, and KEGG databases using BLASTX to identify the proteins that had the highest sequence similarity with the given transcripts to retrieve their function annotations and a typical cut-off E-values less than $1.0 \times 10^{-5}$ was set. BLAST2GO (http://www. blast2go.com/b2ghome) program was used to get GO annotations of unique assembled transcripts for describing biological processes, molecular functions and cellular components. Metabolic pathway analysis was performed using the Kyoto Encyclopedia of Genes and Genomes (KEGG, http://www.genome.jp/kegg/).

\section{Identification of differentially expressed genes}

To identify differentially expressed ROS genes (DERGs) between control and samples treated with trypsin, the expression level of each transcript was calculated according to the fragments per kilobase of exon per million mapped reads (FRKM) method. Differential expression analysis was performed by EdgeR software from the $\mathrm{R}$ statistical package. The false discovery rate (FDR) was used to adjust the resulting $P$-values using the Benjamini and Hochberg approach.

\section{GO and KEGG enrichment for differentially expressed genes}

Functional-enrichment analyses including GO and KEGG were performed as reported by Candar-Cakir et al. [21]. The enriched GO terms were shown with DAGs (directed acyclic hierarchical graph) and bar charts [21].

\section{Gene expression analysis by reverse transcription-qPCR}

Total RNA was extracted as described above. Reverse Transcription-qPCR was performed as reported by Yang et al. [22]. The information of primers is listed in Table 6. $\beta$-actin was used as internal control. The relative copy numbers of the genes were obtained by the $2^{-\Delta \Delta C t}$ method $[10,22]$.

\section{Protein-protein interaction (PPI) analyses PPI network generation}

The ROS related proteins were screened from the ISanger cloud platform and imported into Cytoscape software. The relationship between ROS related proteins and their putative targets was visualized through PPI networks of ROS, DERGs, or Hubs of H. undatus induced by trypsin using Cytoscape [19].

\section{Network topological parameters}

Several network topological parameters could compare and characterize the complex networks. The primary topological parameters of networks were calculated by NetworkAnalyzer [19]. Here, the edges were considered as undirected. The equation $y=\beta x^{\alpha}$ and parameters $R^{2}$ and correlation described the fit to the power line [23].

\section{Module}

A Molecular Complex Detection (MCODE) analysis was performed to identify the clusters in the entire ROS related network (Degree cutoff: 2; Node score cutoff: 0.2; KCore: 2; Max depth: 100) [24]. Here, the edges were treated as directed for MCODE or next hub node analysis.

\section{Analysis of hubs}

The key genes in the PPI network were investigated topologically by NetworkAnalyzer. The cytoHubba plugin of Cytoscape further analyzed the network, and the high degree nodes were identified [25]. Through the cytoHubba plugin, 11 topological analysis methods were obtained. To increase the sensitivity and specificity, besides three centrality methods including degree, closeness, and betweenness centrality, the top 20 hubforming proteins were also identified based on local method, including MCC, MNC and DMNC, respectively. Then, the overlapping proteins were considered as the hubs. Finally, we found the common nodes using Venn diagrams [26]. 
Determination of the weight loss rate

The weight loss rates for each group were determined using 3 replicates $(n=6)$ and were recorded at the same time every day for 9 days as the fruit was stored at $25^{\circ} \mathrm{C}$. The replicate information, including recording time point or temperature, among others, was identical to that of other index determinations, including browning index and electrical conductivity. The percentage of weight loss was calculated after days of storage.

\section{Quantification of lipid peroxides in $H$. undatus peel}

MDA contents were measured using the thiobarbituric acid reactive substrates (TBARS) assay as reported by Zhou et al. (2014) [27].

\section{Determination of superoxide and hydrogen peroxide content of $\mathrm{H}$. undatus peel}

First, $2 \mathrm{~g}$ of $H$. undatus peel was ground with $6 \mathrm{ml} 50$ $\mathrm{mM}$ PBS $(\mathrm{pH}=7.8)$ and $1 \%(\mathrm{w} / \mathrm{v})$ PVP at $0{ }^{\circ} \mathrm{C}$. The sample was obtained by $12,000 \mathrm{~g}$ centrifugation $\left(4{ }^{\circ} \mathrm{C}, 15\right.$ min). The production of $\mathrm{O}_{2}{ }^{-}$and hydrogen peroxide $\left(\mathrm{H}_{2} \mathrm{O}_{2}\right)$ was as described by Schneider et al. [28] or $\mathrm{Li}$ and Imlay in 2018 [29], respectively.

\section{Statistical analyses}

The SPSS statistical software package (11.0.1) (15 November 2001, SPSS Inc., Chicago, IL) was used for data analyses. Our results were obtained by 3 independent experiments. A paired sample t-test was used to analyze the differences between samples. Significant difference was estimated by $p<0.05$. Highly significant means $p<$ 0.01 .

\section{Supplementary information}

Supplementary information accompanies this paper at https://doi.org/10. 1186/s12864-020-06850-1.

Additional file 1: Fig. S1. Statistics of assembly length of transcripts. Fig. S2. Venn diagram of sequence statistics of functional annotation of RNA-Seq data for each database. Fig. S3. COG and NOG classification of transcripts or unigenes. Fig. S4. GO classification of unigenes. a Bar chart of three categories; (b-d) Molecular Function (MF), Biological Process (BP), or Cellular Component (CC) categories of unigenes. Fig. S5. Histogram of KEGG terms associated with trypsin. Different categories of KEGG terms were shown in different color. Red, Metabolism; Green, Genetic Information Processing; Purple, Environmental Information Processing; Blue, Cellular Processes; Yellow, HD, Organismal Systems; Brown, Human Diseases. Fig. S6. Relationships between GO terms of downregulated DERGs in a Directed Acyclic Graph (DAG). The information of color and arrow was same to that in Fig. 3. Fig. S7. Relationships between $\mathrm{GO}$ terms of upregulated DERGs in a Directed Acyclic Graph (DAG). The information of color and arrow was same to that in Fig. 3. Fig. S8. PPI network of two patterns of ROS related genes by cytoscape. a Upregulated; b Downregulated. Fig. S9. PPI network of two patterns of DERGs by cytoscape. a Upregulated; b Downregulated. Fig. S10. Power law distribution of node degree. a Degree distribution of ROS related PPI network; b Degree distribution of the DERGS PPI subnetwork. The graph displays a decreasing trend of degree distribution, with increasing number of links displaying scale-free topology. Black, red or blue curves represent total, upregulated, and downregulated PPI subnetworks, respectively. Fig. S11. RNA-seq analysis of 10 hub genes of DERGs of $H$. undatus peel with or without trypsin at $159 \mathrm{~h}$ of storage and RT-qPCR confirmation.

Additional file 2: Fig. S12. KEGG pathway of map 00940. Significant expressed genes were highlighted with blue borders.

Additional file 3: Fig. S13. KEGG pathway of map 04016. Significant expressed genes were highlighted with blue borders.

Additional file 4: Table S1. Number of transcripts or unigenes in 6 public databases.

Additional file 5: Table S2. Common unigenes in public databases including NR, Swiss-Prot, Pfam, COG, GO and KEGG.

Additional file 6: Table S3. Unigenes in functional groups based on COG and NOG classifications.

Additional file 7: Table S4. Number of unigenes enriched in KEGG pathways.

Additional file 8: Table S5. Information of unigenes shown in the volcano plot spectrum in Fig. 2a.

Additional file 9: Table S6. Common unigenes in different gene sets. All of the significantly expressed genes are named as allsignificant1703; 1117 ROS related genes are named as ROSall1117, including 434 upregulated and 465 downregulated genes; 85 DERGs are named as ROSsig85.

Additional file 10: Table S7. GO terms of DERGs by trypsin. Additional file 11: Table S8. Full list of ROS related genes in each pattern after treatment with trypsin.

Additional file 12: Table S9. PPI network parameters of ROS related genes by Cytoscape.

Additional file 13: Table S10. PPI network parameters of upregulated ROS related genes by Cytoscape.

Additional file 14: Table S11. PPI network parameters of downregulated ROS related genes by Cytoscape.

Additional file 15: Table S12. Clusters of PPI network of ROS related genes by MCODE.

Additional file 16: Table S13. Top20 genes in DERGs network ranked by methods of MCC, degree, closeness or betweenness.

Additional file 17: Table S14. PPI network parameters of hub genes by Cytoscape.

Additional file 18: Table S15. Annotation of hub nodes of DERGs PPI network.

Additional file 19: Table S16. Information of hub genes

\section{Acknowledgements}

We are grateful for the free online Majorbio I-Sanger Cloud Platform (www.isanger.com). We would also like to thank English Editing by Elsevier Language Editing Services (Registration No. 331566771)

\section{Authors' contributions}

$X \mathrm{~L}$ and $\mathrm{YY}$ conceived the projects. $\mathrm{XL}$ and $\mathrm{XYP}$ designed and executed the experiments. $\mathrm{HCY}$ and $Y X Y$ contributed expertise in the $H$. undatus system, XRL and BRL detected the activities of enzymes, and XYP and BRL detected the browning indices and analyzed the data. All authors discussed the results. $\mathrm{XL}$ and $\mathrm{XYP}$ cowrote the paper. All authors have read and approved the manuscript.

\section{Funding}

This work has been supported by the National Key Research and Development Program of China (2017YFC1600802). XL is supported by the State Key Laboratory of Cotton Biology Open Fund (CB2018A22), and the Natural Science Foundation of Henan Province of China (182300410083). XYP is supported by the Natural Science Program of the Educational Commission of Henan Province of China (18A310013). Publication costs for this work were funded the Science and Technique Foundation of Henan Province (182102310644) 


\section{Availability of data and materials}

All data and materials used in this research are publicly available. Raw sequence data from this study have been submitted to the NCBI sequence read archive under the BioProject accession [PRJNA509494] and are available at the following link: https://trace.ncbi.n/m.nih.gov/Traces/sra_sub/sub. cgi?acc=SRP173572. Other supporting data are included as additional files listed below and are submitted with the manuscript.

\section{Ethics approval and consent to participate}

Not applicable.

\section{Consent for publication}

Not applicable.

\section{Competing interests}

None of the authors have potential financial or ethical conflicts of interest with the contents of this submission.

\section{Author details}

${ }^{1}$ College of Food and Bioengineering, Henan University of Science and Technology, No. 263, Kaiyuan Avenue, Luolong District, Luoyang city 471023, Henan, China. ${ }^{2}$ State Key Laboratory of Cotton Biology, Henan University, Kaifeng 455000, China. ${ }^{3}$ Key Laboratory of Desert and Desertification, Chinese Academy of Sciences, Lanzhou 730000, Gansu, China. ${ }^{4}$ Ministry of Education Key Laboratory of Cell Activities and Stress Adaptations, Lanzhou University, Lanzhou 730000, China. ${ }^{5}$ College of Medical Technology and Engineering, Henan University of Science and Technology, Luoyang 471023, China.

Received: 24 August 2019 Accepted: 18 June 2020

Published online: 26 June 2020

\section{References}

1. Fan QJ, Yan FX, Qiao G, Zhang BX, Wen XP. Identification of differentially-expressed genes potentially implicated in drought response in pitaya (Hylocereus undatus) by suppression subtractive hybridization and cDNA microarray analysis. Gene. 2014;533: 322-31. https://doi.org/10.1080/14620316.2011.11512740.

2. Freitas STD, Mitcham EJ. Quality of pitaya fruit (Hylocereus undatus) as influenced by storage temperature and packaging. Sci Agric. 2013;70(4): 257-62. https://doi.org/10.1590/\$0103-90162013000400006.

3. Yang ZQ, Zhong XM, Fan Y, Wang HC, Li JG, Huang XM. Burst of reactive oxygen species in pedicel-mediated fruit abscission after carbohydrate supply was cut off in longan (Dimocarpus longan). Front Plant Sci. 2015;6: 360. https://doi.org/10.3389/fpls.2015.00360.

4. Lu Z, Sethu R, Imlay JA. Endogenous superoxide is a key effector of the oxygen sensitivity of a model obligate anaerobe. Proc Natl Acad Sci U S A. 2018:115(14):E3266-75. https://doi.org/10.1073/pnas.1800120115.

5. Li Q, Wei QY, Yuan ED, Yang JG, Ning ZX. Interaction between four flavonoids and trypsin: effect on the characteristics of trypsin and antioxidant activity of flavonoids. Int J Food Sci Tech. 2014;49(4):1063-9. https://doi.org/10.1111/ijfs.12401.

6. Li X, Tang ZC, Zhao CY, Pang XY, Li XL, Liu YH. Trypsin slows the ageing of mice due to its novel superoxide scavenging activity. Appl Biochem Biotech. 2017;181(4):1-12. https://doi.org/10.1007/s12010-016-2301-7.

7. Yu XJ, Chen H, Huang CY, Zhu XY, Wang DS, Liu XY, Sun J, Zheng JY, Li HJ, Wang Z, Wang ZP. Transcriptomic mechanism of a phytohormone 6-benzylaminopurine (6BAP) stimulating lipid and DHA synthesis in Aurantiochytrium sp. J Agric Food Chem. 2019;67:19. https://doi.org/10.1021/acs.jafc.8b07117.

8. Xia CJ, Li SF, Hou WY, Fan ZF, Xiao H, Lu MG, Sano T, Zhang ZX. Global transcriptomic changes induced by infection of cucumber (Cucumis sativus L.) with mild and severe variants of hop stunt viroid. Front Microbiol. 2017;8: 2427. https://doi.org/10.3389/fmicb.2017.02427.

9. Zheng $Y$, Wang $Y$, Ding B, Fei ZI. Comprehensive transcriptome analyses reveal that potato spindle tuber viroid triggers genome-wide changes in alternative splicing, inducible trans-acting activity of phasiRNAs and immune responses. J Virol. 2017;91: e00247-17. https://doi.org/10.1128/MV.00247-17.

10. Xu M, Liu CL, Luo J, Qi Z, Yan Z, Fu Y, Wei SS, Tang H. Transcriptomic de novo analysis of pitaya (Hylocereus polyrhizus) canker disease caused by Neoscytalidium dimidiatum. BMC Genomics. 2019;20:10. https://doi.org/10. 1186/s12864-018-5343-0.

11. Hua QZ, Chen CJ, Chen Z, Chen PK, Ma YW, Wu JY, Zheng J, Hu GB, Zhao JT, Qin YH. Transcriptomic analysis reveals key genes related to betalain biosynthesis in pulp coloration of Hylocereus polyrhizus. Front Plant Sci. 2016; 6:1179. https://doi.org/10.3389/fpls.2015.01179.

12. Azodi MZ, Peyvandi H, Rostami-Nejad M, Safaei A, Rostami K, Vafaee R, Heidari M, Hosseini M, Zali MR. Protein-protein interaction network of celiac disease. Gastroenterol Hepatol Bed Bench. 2016;9(4):268-77.

13. Chin $\mathrm{CH}$, Chen $\mathrm{SH}$, Wu HH, Ho CW, Ko MT, Lin CY. cytoHubba: identifying hub objects and sub-networks from complex interactome. BMC Syst Biol. 2014;8:S11. https://doi.org/10.1186/1752-0509-8-S4-S11.

14. Ren YL, Wang YF, Bi Y. Postharvest BTH Treatment induced disease resistance and enhanced reactive oxygen species metabolism in muskmelon (Cucumis melo L.) fruit. Eur. Food Res Technol. 2012;234(6):96371. https://doi.org/10.1007/s00217-012-1715-x.

15. Duan XW, Liu T, Zhang DD, Su XG, Lin HT, Jiang YM. Effect of pure oxygen atmosphere on antioxidant enzyme and antioxidant activity of harvested litchi fruit during storage. Food Res Int. 2011:44(7):1905-11. https://doi.org/ 10.1016/j.foodres.2010.10.027.

16. Liu YH, Zeng Y, Wang Q, Sun CY, Xi HH. Drying characteristics, microstructure, glass transition temperature, and quality of ultrasoundstrengthened hot air drying on pear slices. J Food Process Pres. 2019;43(3): e13899. https://doi.org/10.1111/jppp.13899.

17. Xi HH, Liu YH, Guo LG, Hu RR. Effect of ultrasonic power on drying process and quality properties of far-infrared radiation drying on potato slices. Food Sci Biotechnol. 2020;29(1):93-101. https://doi.org/10.1007/s10068-019-00645-1.

18. Li X, Liu XR, Yin Y, Yu HC, Zhang M, Jing HN, Ma YC, Xiong XL, Pang XY. Transcriptomic analysis reveals key genes related to antioxidant mechanisms of Hylocereus undatus quality improving by trypsin during storage. Food Funct. 2019;10:8116-28. https://doi.org/10.1039/c9fo00809h.

19. Wu BL, Xie JJ, Du ZP, Wu JY, Zhang PX, Xu LY, Li EM. PPI network analysis of mRNA expression profile of Ezrin knockdown in esophageal squamous cell carcinoma. Biomed Res Int. 2014;651954. https://doi.org/10.1155/2014/651954.

20. Grabherr MG, Haas BJ, Yassour M, Levin IZ, Thompson DA, Amit I, Adiconis X, Fan L, Raychowdhury R, Zeng QD, Chen ZH, Mauceli E, Hacohen N, Gnirke A, Rhind N, Palma FD, Birren BW, Nusbaum C, Lindblad-Toh K, Friedman N, Regev A. Full-length transcriptome assembly from RNA-Seq data without a reference genome. Nat Biotechnol. 2011;29:644-52. https://doi.org/10.1038/nbt.1883.

21. Candar-Cakir B, Arican E, Zhang BH. Small RNA and degradome deep sequencing reveals drought-and tissue-specific micrornas and their important roles in drought-sensitive and drought-tolerant tomato genotypes. Plant Biotechnol J. 2016;14:1727-46. https://doi.org/10.1111/pbi.12533.

22. Yang AM, Yu L, Chen Z, Zhang SX, Shi J, Zhao XZ, Yang YY, Hu DY, Song BA. Label-free quantitative proteomic analysis of chitosan oligosaccharidetreated rice infected with southern rice black-streaked dwarf virus. Viruses. 2017:9:115. https://doi.org/10.3390/v9050115.

23. Wu BL, Li CQ, Zhang PX, Yao QL, Wu JY, Han JW, Liao LD, Xu YJ, Lin RJ, Xiao $D W, X u L Y, L i E M, L i X$. Dissection of miRNA-miRNA interaction in esophageal squamous cell carcinoma. PLoS One. 2013;8(9):e73191. https:// doi.org/10.1371/journal.pone.0073191.

24. Vig S, Talwar P, Kaur K, Srivastava R, Srivastava AK, Datta M. Transcriptome profiling identifies p53 as a key player during calreticulin deficiency: implications in lipid accumulation. Cell Cycle. 2015;14(14):2274-84. https:// doi.org/10.1080/15384101.2015.1046654.

25. Li Y, Cai ZH, Zhu BA, Xu CS. Identification of key pathways and genes in the dynamic progression of HCC based on WGCNA. Genes. 2018;9:0. https://doi. org/10.3390/genes9020092.

26. Huang HJ, Luo BB, Wang BQ, Wu QW, Liang YM, He Y. Identification of potential gene interactions in heart failure caused by idiopathic dilated cardiomyopathy. Med Sci Monit. 2018;24:7697-709. https://doi.org/10.12659/MSM.912984.

27. Zhou Q, Ma C, Cheng SC, Wei BD, Liu XY, Ji SJ. Changes in antioxidative metabolism accompanying pitting development in stored blueberry fruit. Postharvest Biol Tec 2014;88:88-95. https:/doi.org/10.1016/.jposthanbio.2013.10.003.

28. Schneider K, Schlegel HG. Production of superoxide radicals by soluble hydrogenase from Alcaligenes eutrophus H16. Biochem J. 1981;193:99-107. https://doi.org/10.1042/bj1930099.

29. Li X, Imlay JA. Improved measurements of scant hydrogen peroxide enable experiments that define its threshold of toxicity for Escherichia coli. Free Radic Biol Med. 2018;120:217-22. https://doi.org/10.1016/.j.reeradbiomed.2018.03.025.

\section{Publisher's Note}

Springer Nature remains neutral with regard to jurisdictional claims in published maps and institutional affiliations. 